图像的对应局部差值图像. 图 4(b) (g) 分别对应于 ACIF, VMF, DDF, DMVF, ANNF 和 $\propto$ TMF. 比较其他滤波器, 图 4(b) 显示了 ACIF 滤波后的差值图像幅度最小, 这意味着噪声图像经 ACIF 滤波后的图像清晰度最好.

\title{
4 结论
}

非线性矢量滤波器对脉冲噪声的滤波性能较好,而线性滤波器则对 Gauss 噪声的抑制效 果更佳. 因此两者的有机结合可为图像处理提供性能稳健的滤波器. 本文提出的自适应彩色 图像滤波器( ACIF) 结合了两者的优点, 通过对彩色图像中噪声程度及类型的分析, 选择合适有 效的局部自适应滤波器进行图像增强. 实验结果显示, ACIF 在滤除脉冲噪声、Gauss 噪声及其 混合噪声以及图像边缘与细节保持方面具有较为稳健的良好性能, 其滤除效果总体上优于 VMF, DDF, DMVF, ANNF 和 $\propto-T M F$ 等彩色图像滤波器.

致谢 本工作为浙江省自然科学基金( 批准号: 698064) 资助项目.

\section{参 考 文 献}

1 Astola J, Haavisto P, Neuvo Y. Vector median filter. Processings of the IEEE, 1990, 78: 678 689

2 Trahanias P E, Venetsanopoulos A N. Vector directional filters A proposed class of multichannel image processing filters. IEEE Trans Image Processing, 1993, 2: 528 534

3 Trahanias P E, Pitas I, Venetsanopoulos A N. Directional processing of color image: Theory and experimental results. IEEE Trans Image Processing, 1996, 5: 868 880

4 Karakos D G, Tranias P E. Combining vector median and vector directional filters: the directional-distance filters. Proc of IEEE Int Conf On Image Processing 95, 1995, 1: 171 174

5 Plataniotis K N, Androutsos D, Venetsanopoulos A N. Content-based color image filters. Electonics Letters, 1997, 33: 203 212

6 Plataniotis K N, Androutsos D, Sri V, et al. Nearest-neighbour multichannael filter. Electonics Letters, 1995, 31: 1910 1911

(1999-04-23 收稿, 1999-07-08 收修改稿)

\section{由 Lagrange 实验得到酚醛玻璃钢的 动态本构方程}

\section{尚嘉兰白以龙徐素珍 (4) 蔡小烨}

( 中国科学院力学研究所, 北京 100080; (4)中国航天工业总公司一院十四研究所, 北京 100076)

摘要 介绍由 Lagrange 实验、分析及拟合, 得到未知材料的动态本构方程的方法. 实验在轻气 炮上进行, 测量多点应力历程, 经 Lagrange 分析得到数值本构关系, 再拟合得到可供设计计算 使用的动态本构方程. 给出酚醛玻璃钢受冲击载荷时, 一维应变状态下的一个本构方程. 方 程计算值与实测结果一致.

\section{关键词 冲击加载 动态本构方程 Lagrange 分析}

动态本构关系是冲击载荷作用下材料力学性能的基本表征, 尤其是应变率在 $10^{3} \sim$ $10^{5} \mathrm{~s}^{-1}$, 应力在 $10^{2} \sim 10^{3} \mathrm{MPa}$ 的动态本构方程, 是抗冲击结构设计和计算中所必须的. 建立动 态本构关系的传统方法是半经验半理论方法，即参考已有的理论, 如 Marlven 的过应力模型、 
Perzyna 的粘塑性模型及 Bodner 和 Partam 的弹粘塑性模型等 ${ }^{[1,2]}$, 经过若干项实验, 确定模型中 的若干个待定参数. 然而对于酚醛玻璃钢这类新型材料, 要预先假定本构模型是很困难的. 所以我们利用力学所的平板碰撞和多点应力测量的 Lagrange 实验技术 ${ }^{[3]}$. 对实验记录进行 Lagrange 分析, 获得了酚醛玻璃钢一维应变动态数值本构关系 ${ }^{[4]}$. 在此基础上讨论了材料本 构的物理特征, 如应力-应变关系的滞回效应、应变率效应和应变、应变率历史效应等, 找到了 能用应变和应变率表达的率型量 一模量函数, 拟合得到揭示出该材料本构特性的可供使用 的完整动态本构方程.

\section{1 实验和测试记录的分析}

试验材料为玻璃纤维增强酚醛树脂复合材料(GFRP), 或称酚醛玻璃钢, 其密度 $\rho_{\mathrm{e}}=1640$ $\mathrm{kg} / \mathrm{m}^{3}$, 波速 $3140 \mathrm{~m} / \mathrm{s}$, 静态拉伸强度 5.7 8.5 MPa, 沿铺层方向拉伸弹模 $20.4 \mathrm{GPa}$, 每毫米厚 度有 5 层铺层.

平板对称碰撞试验在中国科学院力学研究所的 $101 \mathrm{~mm}$ 口径轻气炮上进行. 实验装置示 于图 1(a) 中. 用自行研制的碳膜压阻传感器监测应力历史, 传感器置于靶板内的不同位置, 见图 1(b). 撞击速度的范围为 $170 \sim 530 \mathrm{~m} / \mathrm{s}$. 典型的应力历史波形示于图 1(c).

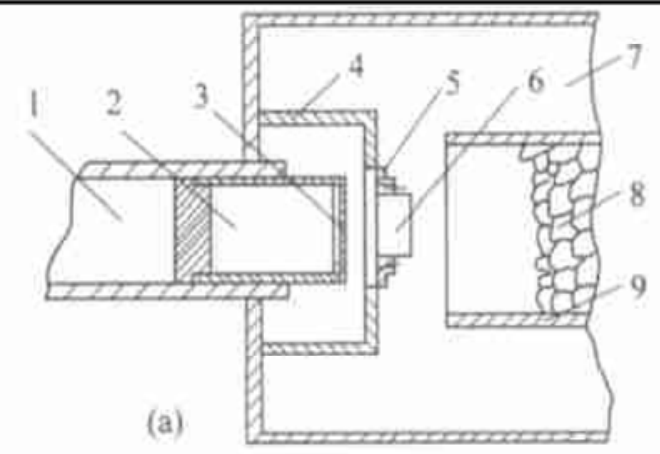

图 1 平板碰撞实验装置( a) 、压力传感器在靶试件中的位置(b) 和测得的应力历史波形 (c)

(b) 6.1 为环氧树脂, $6.2 \sim 6.5$ 分别为应力探头 $1(k=1), 2(k=2), 3(k=4)$ 和 $4(k=6), 6.6$ 为探针

采用 70 年代 Fowles ${ }^{[5]}$, Williams 和 Cowperthwaite ${ }^{[6]}$ 提出, 后经 Grady ${ }^{[7]}$ 和 Seaman ${ }^{[8]}$ 完善了的 Lagrange 分析方法, 对试验记录进行处理. 它在无需任何本构假定的前提下, 对一次试验得到 的多个应力历史波形, 用连续方程和运动方程直接计算出材料内部的力学量和变形量. 这是 力学上解逆问题的一种可行方法, 而且其结果能更客观真实地反映材料自身的物理本质 ${ }^{[9]}$.

分析结果由图 2 给出. 图 2(a) 给出典型的应力、应变率随应变的变化. 图 2(b) 给出 6 次 试验的压应力与体应变的关系. 图中 $k=2$ 表示在第 2 个探头位置的质点.

2 酚醛玻璃钢动态本构关系的特征

图 2 中的 $\sigma \varepsilon$ 曲线描述了材料中的一个微元在实验过程中所经历的过程. 在图 2 中可以 看到, GFRP 的 $\sigma \varepsilon$ 曲线在加载段上凸, 而在卸载段上凹. 在图 2 (a) 中, 当 $\varepsilon$ 达到 $30 \times 10^{3} \mu \varepsilon$ 时, 应力 - 应变曲线向应变轴方向弯曲, 应变率 - 应变线并未继续下降, 而是在低应变率处徘徊, 应变仍不断增加, 从而造成材料进入类似蠕变的状态. $\sigma \varepsilon$ 曲线构成明显的滞回, 而且应变率 的变化控制着滞回的大小. 另外在图 2(a) 中还可看到, 在试验过程中, 随着应变的增加,一个 质点首先达到应变率峰值 $\varepsilon_{\mathrm{m}}$, 之后应力、应变逐次达到其峰值 $\sigma_{\mathrm{m}}$ 和 $\varepsilon_{\mathrm{m}}$. 各次试验的应变率值 

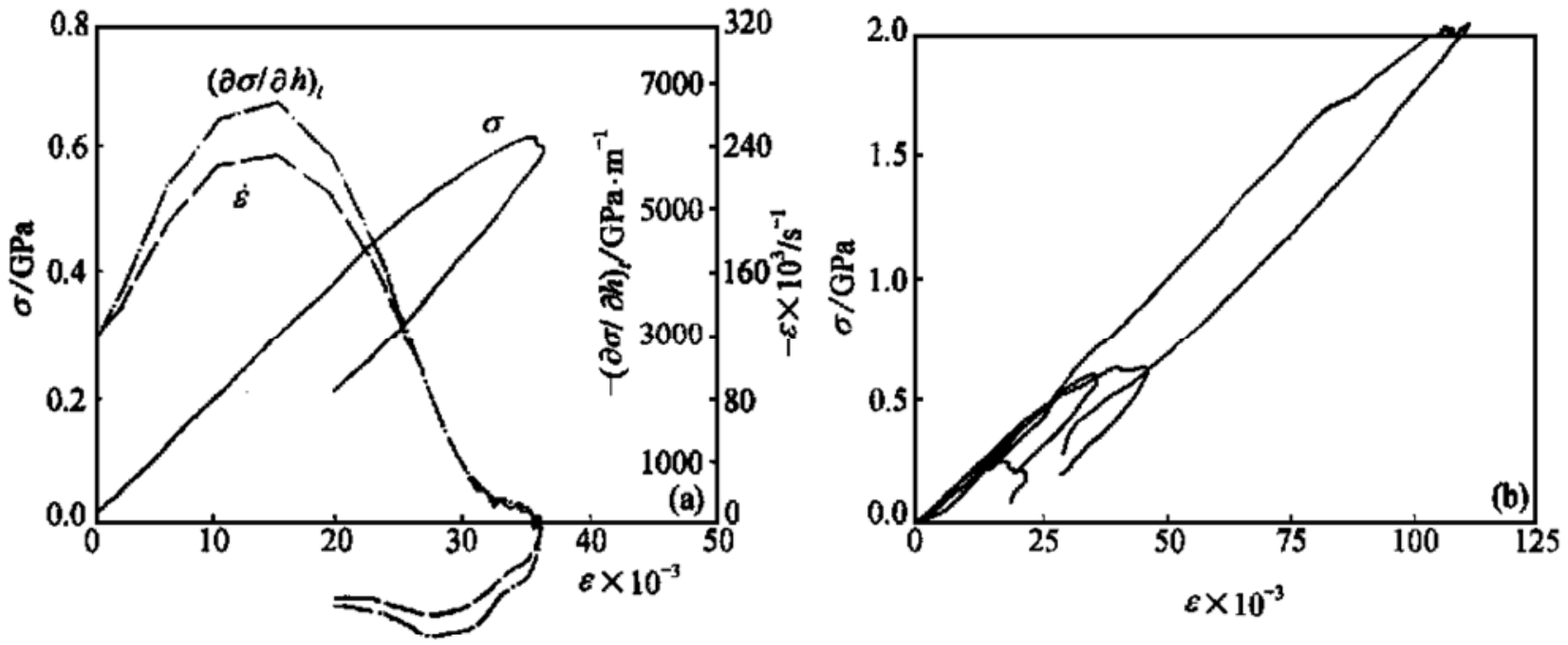

图 2 Lagrange 分析的结果

(a) 应力、应力梯度和应变率-应变关系; (b) 6 个不同加载率试验的应力-应变关系. $k=0$, No. 87-102

从 $40 \times 10^{3} \mathrm{~s}^{-1}$ 变到 $385 \times 10^{3} \mathrm{~s}^{-1}$, 比值 $\varepsilon_{\mathrm{m}} / \sigma_{\mathrm{m}}$ 和 $\varepsilon_{\mathrm{m}} / \varepsilon_{\mathrm{m}}$ 的最大变化分别为约 $10 \%$ 和 $20 \%$. 这 表明不同加载率的试验使应变率变化一个量级 时, 应力峰值和应变峰值也有一个量级的变化. 说明 $\sigma_{\mathrm{m}}$ 和 $\varepsilon_{\mathrm{m}}$ 与材料在历史上曾经经历过的应变 率水平密切相关.

用 $R=\left.\frac{\mathrm{d} \sigma}{\mathrm{d} \varepsilon}\right|_{h}$ 表示 $\sigma \varepsilon$ 曲线的斜率, 可以发现 一个质点的波动经历可以用 $R-\varepsilon$ 曲线 (图 3) 给 出. 在加载初期, $\varepsilon$ 由零开始增加, $R$ 值较高且变 化不大. 随着 $\varepsilon$ 的增加, $R$ 逐渐降低. 在应力峰 值处, $R=0$. 这之后在应力下降而应变仍在增加 的阶段, $R$ 下降越来越快, 在 $\varepsilon=\varepsilon_{\mathrm{m}}$ 处, $R=-\infty$.

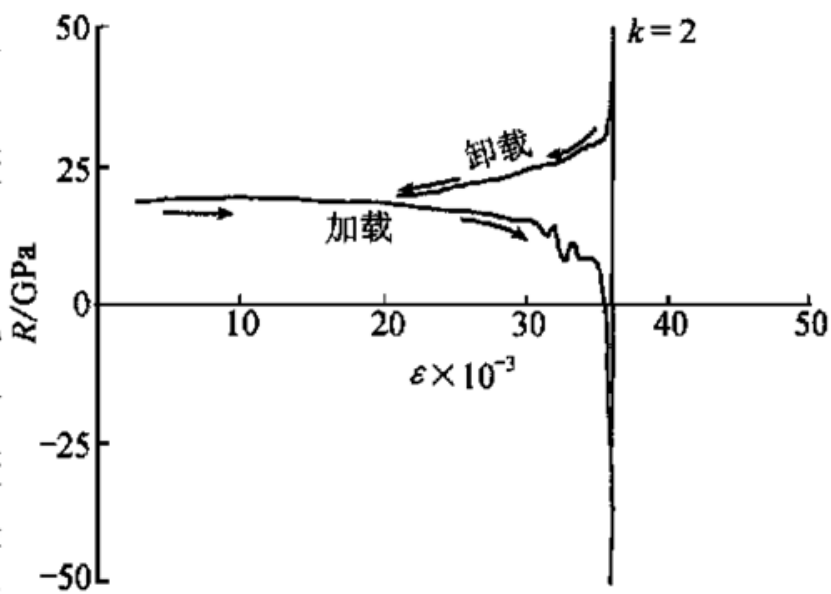

图 3 模量函数 $R$ 和体应变 $\varepsilon$ 的关系 然后在应力、应变均下降的卸载段, $R$ 由一个很高的正值(或+ $\infty$ ), 迅速下降而趋于一个有限 值. 该值通常高于加载初期的 $R$ 值.

总之, GFRP 的动态本构关系有如下特点: (1) 材料经受冲击载荷过程中, 应力、应变和应 变率都是随时间而变化的. (2) 材料的瞬态应力不仅与当时的瞬态应变、瞬态应变率有关, 而 且与应变和应变率的历史有关. 这种明显的非平衡效应表明, 不存在 $\sigma$ 与 $\varepsilon$ 和 $\varepsilon$ 的简单代数 表达式. (3) 分析材料受冲击载荷的波动过程中的 $\sigma, \varepsilon$ 和 $\varepsilon$ 关系, 可以认为解决问题的可能 途径是考察率型量间的关系.

\section{3 模量函数 $\boldsymbol{R}(\varepsilon, \varepsilon)$}

由于 GFRP 各力学量间的关系有明显的非平衡效应和滞回效应, 考察一个率型量: 模量函 数. 令模量函数为

$$
R(\varepsilon, \varepsilon)=\left.\frac{\mathrm{d} \sigma}{\mathrm{d} \varepsilon}\right|_{h}=\frac{\dot{\sigma}}{\dot{\varepsilon}},
$$

$R(\varepsilon, \varepsilon)$ 是 $\sigma \varepsilon$ 曲线的斜率, 也是应力率 $\sigma$ 与应变率 $\varepsilon$ 的比值, 它是材料刚度的标志. 图 3 给出 
一个微元在波动过程中 $R$ 随 $\varepsilon$ 的变化. 图 3 中 $\varepsilon$ 较大的加载段, $R$ 迅速下降, 表明材料模量损 失、刚度下降而呈现软化特性.

考察多次试验的 $R$ 与 $\varepsilon$ 和 $\varepsilon$ 的关系可以看到, 对于不同的 $\varepsilon, R$ 随 $\varepsilon$ 的变化趋势大致相同, 图 4(a) 和(b) 分别对应应变加载和卸载阶段. 在图 4(a) 中, 当 $\varepsilon$ 较大时, $R$ 趋于一个饱和值 $R_{0}$. 8 下降时, $R$ 下降由缓慢到迅速; 当 $\varepsilon$ 趋于零时, $R$ 趋于很大的负值. 在 $\varepsilon=20 \times 10^{3} \mathrm{~s}^{-1}$ 附 近, 不同 $\varepsilon$ 的 $R-\varepsilon$ 曲线略有不同. 在应变卸载段, $R-\varepsilon$ 也有类似的趋势, 见图 $4(\mathrm{~b})$.

可以认为, 在应变加载和卸载阶段, $R$ 是主要依赖于 $\varepsilon$ 而随 $\varepsilon$ 略有不同的两族双曲线. 其 表达式可设为

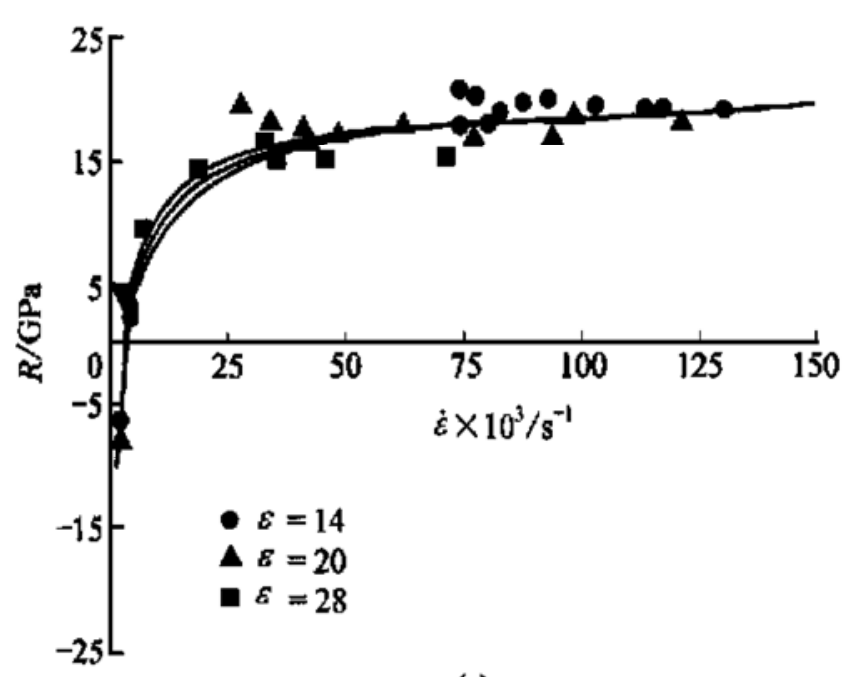

(a)

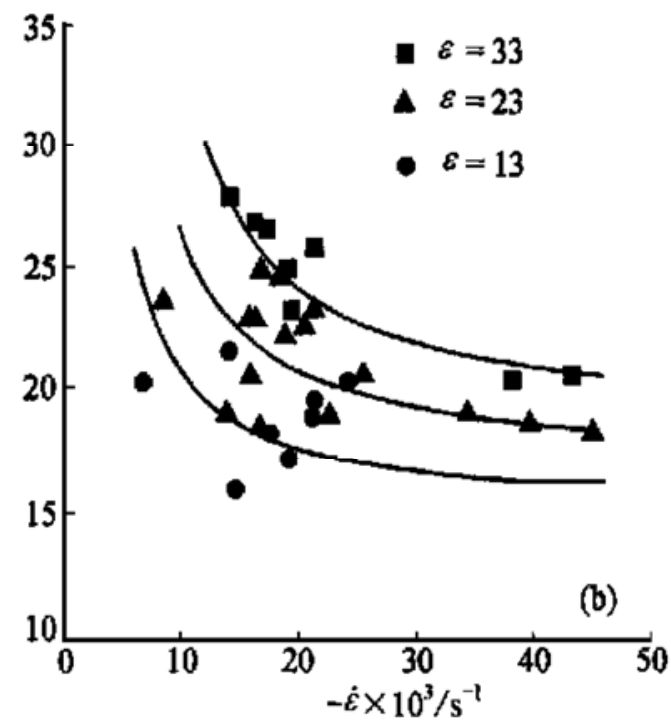

图 4 应变相同的 $R$ 值随 $\varepsilon$ 的变化

(a) $d \varepsilon>0 ;$ (b) $d \varepsilon<0$

$$
\begin{gathered}
R=R_{0}\left[1-A(\varepsilon)\left(\frac{\varepsilon}{\varepsilon_{*}}\right)^{-m}\right], \quad \mathrm{d} \varepsilon>0, \\
R=R_{u 0}\left[1+R_{u 1} \varepsilon+B(\varepsilon)\left(-\frac{\varepsilon}{\varepsilon_{*}}\right)^{-n}\right], \quad \mathrm{d} \varepsilon<0,
\end{gathered}
$$

其中 $\varepsilon_{*}=10^{3} \mathrm{~s}^{-1} \varepsilon$. 由实验数据拟合得到 $R_{0}=22.5 \mathrm{GPa}, R_{u 0}=13.5 \mathrm{GPa}, R_{u 1}=12.1, m=$ $0.55, n=1.44$.

考察 $A(\varepsilon)$ 和 $B(\varepsilon)$ 随 $\varepsilon$ 的变化, 可用三次幂级数拟合得到其各项系数:

$$
\begin{aligned}
& A(\varepsilon)=\sum_{i=0}^{3} A_{i} \varepsilon, \\
& B(\varepsilon)=\sum_{i=0}^{3} B_{i} \dot{\varepsilon},
\end{aligned}
$$

其中 $A_{0}=0.911, A_{1}=0.902 \times 10^{2}, A_{2}=-1.85 \times 10^{3}, A_{3}=7.69 \times 10^{3}, B_{0}=-5.48, B_{1}=1.36$ $\times 10^{3}, B_{2}=-1.16 \times 10^{4}, B_{3}=3.48 \times 10^{4}$.

注意图 3 中 $R<0, \varepsilon$ 趋于零部分, 它与 $\sigma \varepsilon$ 线上应力达到峰值后逐渐下降而应变仍在增加 部分相对应. 实验中材料内传播的是高速冲击引起的强应力波. 当材料进入波动状态, 其应 变率就达到 $10^{3} \sim 10^{4} \mathrm{~s}^{-1}$. 方程(2) 和(3) 在这类条件下成立. 
4 酚醛玻璃钢的动态本构方程

式(2)和(3) 描述材料受平面波冲击加载时质点经历波动过程中模量函数的变化. 由式 (1) 得

$$
\dot{\sigma}=R(\varepsilon, \varepsilon) \varepsilon
$$

于是 GFRP 的微分型本构方程为

$$
\begin{array}{ll}
\sigma=R_{0}\left[1-A(\varepsilon)\left(\frac{\varepsilon}{\varepsilon_{*}}\right)^{-m}\right] \varepsilon, & \mathrm{d} \varepsilon>0, \\
\sigma=R_{u 0}\left[1+R_{u 1} \varepsilon+B(\varepsilon)\left(-\frac{\varepsilon}{\varepsilon_{*}}\right)^{-n}\right] \dot{\varepsilon} & \mathrm{d} \varepsilon<0 .
\end{array}
$$

若初始时刻 $t=0, \sigma=0, \varepsilon=0$, 那么任何一点的应力表达式为

$$
\begin{gathered}
\sigma=R_{0}\left|\varepsilon-\int_{0}^{\varepsilon} A(\varepsilon)\left(\frac{\varepsilon}{\varepsilon_{*}}\right)^{-m} \mathrm{~d} \varepsilon\right|, \quad \mathrm{d} \varepsilon>0, \\
\sigma=\sigma\left|\varepsilon=\varepsilon_{\mathrm{m}}+R_{u 0}\right|\left(\varepsilon-\varepsilon_{\mathrm{m}}\right)+\frac{R_{u} 1}{2}\left(\varepsilon^{2}-\varepsilon_{\mathrm{m}}^{2}\right)+\int_{\varepsilon_{\mathrm{m}}}^{\varepsilon} B(\varepsilon)\left(-\frac{\varepsilon}{\varepsilon_{*}}\right)^{-n} \mathrm{~d} \oint, \quad \mathrm{d} \varepsilon<0, \text { (8b) }
\end{gathered}
$$

其中 $\varepsilon_{\mathrm{m}}$ 是在 $\varepsilon=0$ 时 $\varepsilon$ 的最大值. 式 $(8 \mathrm{a})$ 中右边第一项是线性项. 第二项可看作是由于材料 在波动过程中经历较大应变而呈现的软化和高应变率历程造成的硬化的综合结果. 式( $8 \mathrm{a})$ 的系数 $R_{0}$ 是材料在高应变率加载下模量 $R$ 的饱和值. 当 $\varepsilon=\infty$ 时, $R=R_{0}$, 波的传播速度为

$$
C\left(\varepsilon_{\infty}\right)=\sqrt{\frac{R_{0}}{\beta_{0}}}=3.70 \mathrm{~km} / \mathrm{s} .
$$

可以认为 $C\left(\varepsilon_{\infty}\right)$ 是 GFRP 受到高应变率加载时, 一维应变平面波的极限传播速度. 在我 们的实验测量中, 最高的波速为 $3.65 \mathrm{~km} / \mathrm{s}$.

将拟合求得的本构方程计算的应力-应变曲线与实测值比较, 两者符合很好. 图 5(a) 给出 这一比较. 令拟合值对实测值的相对百分误差为 $E R$. 除加载初期几点误差较大 $(E R<$ $\pm 20 \%$ ) 之外, 在应变加载段 $(\mathrm{d} \varepsilon>0$ ), 平均误差 $E R<8.83 \%$. No. 87-102和87-113符合得尤其

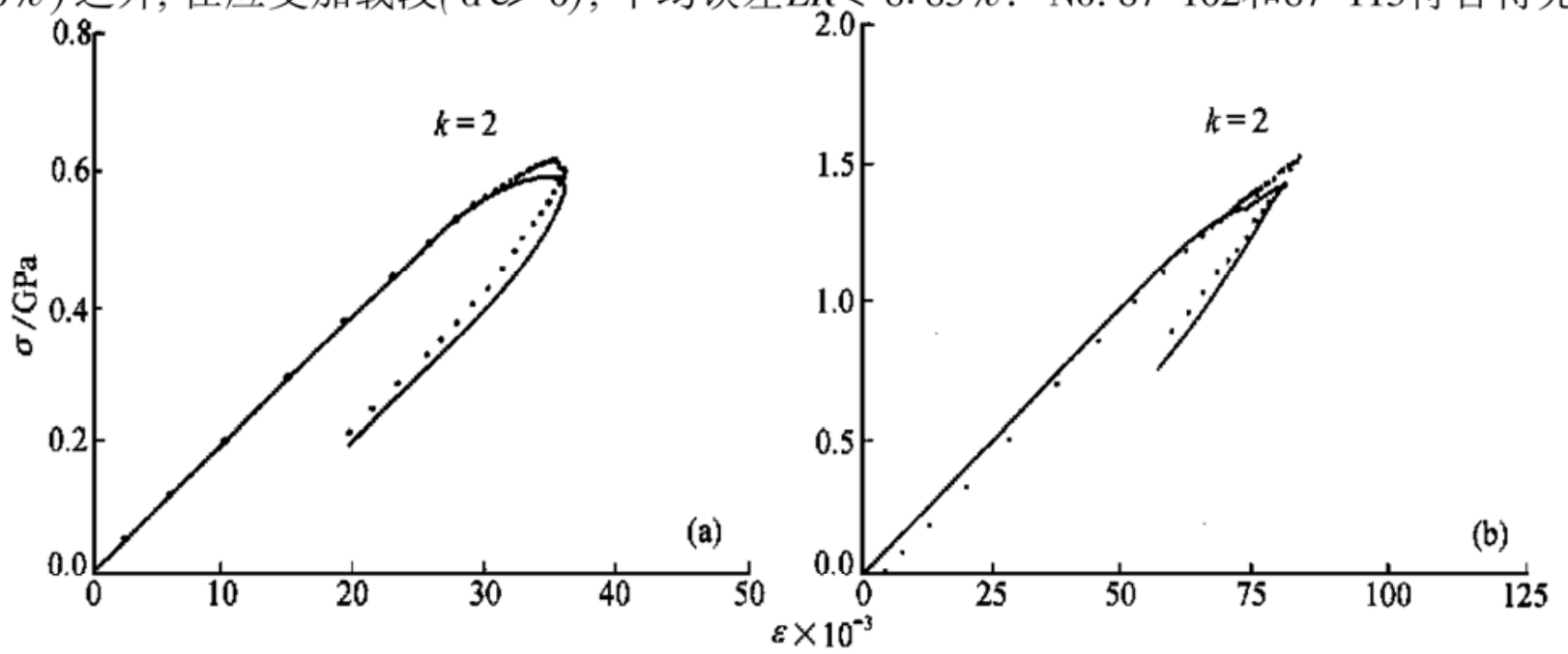

图 5 应力-应变关系拟合计算值 (实线) 与实验值(点线)的比较

(a) No. 87-102; (b) 未参加拟合本构关系的试验. No. 88-59 
好, 平均误差 $E R \leqslant \pm 2.1 \%$, 最大误差 $E R \leqslant 4.3 \%$. 由于误差积累, 卸载段误差略大, 但拟合线 与实测线几乎平行, 两者斜率吻合较好. 图 5(b) 给出一次未参与拟合本构方程的试验, 拟合 线与实测线亦符合较好.

本方程的适用范围由试验条件为 (1) 初始应变率 $\varepsilon>10^{3} \mathrm{~s}^{-1}$; (2) 应力幅值 $\sigma \leqslant 2.2 \mathrm{GPa}$; (3) 应变幅值 $\varepsilon \leqslant 0.11$.

\section{5 结论}

本文给出了酚醛玻璃钢受到冲击载荷时, 在一维应变状态下的一个本构方程. 它是由多 点实测应力历史, 经过 Lagrange 分析得到的数值本构关系拟合得到的. 本方程充分反映了 GFRP 的滞回效应、应变率效应和应变、应变率历史效应. 方程计算值与实测结果吻合较好.

致谢 本工作为国家自然科学重点基金( 批准号: 19132030) 资助项目.

\section{参 考 文 献}

1 杨桂通, 熊祝华. 塑性动力学. 北京: 清华大学出版社, 1984. 48 79

2 周光泉. 关于无屈服面粘塑性理论. 爆炸与冲击, 1983, 3( 4) : 25 33

3 赵士达, 沈乐天, 赵双录. 用于材料动态性能实验的单级轻气炮. 兵工学报, 1985, 4: 49 54

4 尚嘉兰, 白以龙, 沈乐天, 等. 酚醛玻璃钢动态本构关系的实验研究. 爆炸与冲击, 1990, 10(1): 1 13

5 Fowles G R, Williams R F. Plane stress wave propagation in solid. JAP, 1970, 41(1): 360 363

6 Cowperthwaite M, Williams R F. Determination of constitutive relationships with multiple gauges in nondivergent waves. JAP, 1971,42 (1): $456 \sim 462$

7 Grady D E. Experimental analysis of spherical wave propagation. JGR, 1973, 78( 8) : 1299 1307

8 Seaman L., Lagrange analysis for multiple stress or velocity gages in attenuating waves. JAP, 1974, 45( 10) : 4303 4314

9 唐志平. Lagremge 分析方法及其新进展. 力学进展, 1993, 23(3) : 348 359

\section{CurNi 单相合金的深过冷定向凝固过程研究}

\section{谢发勤 李金山 傅恒志}

(西北工业大学凝固技术国家重点实验室, 西安 710072)

摘要利用过冷度的遗传性, 提出将合金熔体深过冷与传统定向凝固相结合的深过冷定向凝 固(SDS) 技术, 并在自制的实验装置上, 对 SDS 技术进行了探索性研究, 实现了 Cu-Ni 合金的深 过冷定向凝固. 结果表明: (1) SDS 技术获得的定向生长组织与 $\mathrm{LMC}\left(G_{\mathrm{L}}=25 \mathrm{~K} / \mathrm{mm}, V=500\right.$ $\mu_{\mathrm{m}} / \mathrm{s}$ ) 法相当, 且一次枝晶间距约为 $30 \mu_{\mathrm{m}}$, 二次分枝被抑制, 一次枝晶主千挺直、细密, 与试样 轴向偏差约为 $5.8^{\circ} ;(2)$ 建立了描述 SDS 过程过冷度与凝固速率之间的数学模型, 即 $\mathrm{T}-\mathrm{T}$ 模 型, 利用此模型可以解释 SDS 试样上微观组织的演化情况.

\section{关键词 合金 熔体 深过冷 定向凝固 深过冷定向凝固}

传统的定向凝固, 如快速凝固(HRS) 法、液态金属冷却 (LMC) 法以及区域熔化液态金属冷 却 (ZMLMC) 法, 都是在固/液界面前沿液相中温度梯度 $G_{\mathrm{L}}>0$ 的条件下完成其凝固过程, 并最 